\title{
Surgery of the elderly in emergency room mode. Is there a place for laparoscopy?
}

\author{
Maciej Michalik ${ }^{1}$, Natalia Dowgiałło-Wnukiewicz ${ }^{1,2}$, Paweł Lech ${ }^{1}$, Krzysztof Zacharz ${ }^{1}$ \\ ${ }^{1}$ Department of General and Minimally Invasive Surgery, University of Warmia and Mazury, Olsztyn, Poland \\ ${ }^{2}$ Department of Anatomy, University of Warmia and Mazury, Olsztyn, Poland
}

Videosurgery Miniinv 2017; 12 (2): 115-119

DOI: https://doi.org/10.5114/wiitm.2017.66833

\begin{abstract}
Introduction: An important yet difficult problem is qualification for surgery in elderly patients. With age the risk of comorbidities increases - multi-disease syndrome. Elderly patients suffer from frailty syndrome. Many body functions become impaired. All these factors make the elderly patient a major challenge for surgical treatment.

Aim: Analysis of the possibility of developing the indications and contraindications and the criteria for surgical treatment of the elderly based on our own cases. Discussion whether there is a place for laparoscopy during surgery of the elderly in emergency room (ER) mode.

Material and methods: The analysis was performed based on seven cases involving surgical treatment of elderly patients who were admitted to the hospital in emergency room mode. The patients were hospitalized in the General and Minimally Invasive Surgery Clinic in Olsztyn in 2016.

Results: Surgical treatment of elderly patients should be planned with multidisciplinary teams. Geriatric surgery centers should be developed to minimize the risk of overzealous treatment and potential complications. Laparoscopy should always be considered in the case of ER procedures or diagnostics.

Conclusions: Elderly patients should not be treated as typical adults, but as a separate group of patients requiring special treatment. Due to the existing additional disease in the elderly, the frailty syndrome, any surgical intervention should be minimally invasive. The discussion about therapy should be conducted by a team of specialists from a variety of medical fields.
\end{abstract}

Key words: minimally invasive surgery, surgery of old age, surgery of elderly.

\section{Introduction}

An important yet difficult problem is the surgical qualification for surgery in elderly patients. With age the risk of comorbidities increases - multi-disease syndrome. Patients in old age suffer from frailty syndrome $[1,2]$. Many body functions become impaired. All these factors make the elderly patient a major challenge for surgical treatment. The patient cannot be treated simply as an adult, but rather as an "older adult". There are changes in metabolism of drugs, ability to regenerate, and healing of intestinal anastomoses or surgical wounds [3]. The elderly patient is also a challenge for postoperative rehabilitation and cooperation with the treating staff because of the often-difficult contact and intellectual capacity and reduced physical fitness. Compared to that of a 30-year-old person, the velocity of nerve conduction, metabolism, cardiac output, kidney function and breathing capacity greatly decrease in a person over 65 years old. Also, the volume of fluid and organs is also greatly reduced in the elderly [1-3]. This

\footnotetext{
Address for correspondence

Natalia Dowgiałło-Wnukiewicz MD, Department of General and Minimally Invasive Surgery, University of Warmia and Mazury,

2 Michała Oczapowskiego St, 10-719 Olsztyn, Poland, phone: +48 604158 786, e-mail: natalia.dowgiallo@gmail.com
} 
carries numerous clinical implications that should be considered during both diagnostic and therapeutic decisions.

In all areas of surgery, there are accepted indications and contraindications for surgery. Indications and contraindications are well defined in vascular surgery, cardiac surgery, orthopedics and neurosurgery. This applies to surgical oncology, where a contraindication can be a spread of cancer in the TNM classification for the effective yet radical treatment but also the safety of a palliative operation [4]. There are contraindications to bariatric surgery such as alcoholism, drug addiction, active psychosis or lack of understanding of the essence of surgery [5]. Finally there are generally accepted criteria to qualify for operations such as the classification of the American Society of Anesthesiologists (ASA) or numerous comorbid conditions which make it impossible to safely carry out the surgery. Contraindications to surgery may also be active foci of infection or failure within one or multiple organs. When qualifying an elderly person for surgery we should take into consideration the possibility to apply overzealous treatment, which will bring the patient only additional and unnecessary suffering with no chance of success.

\section{Aim}

Analysis of the possibility of developing the indications and contraindications and the criteria for surgical treatment of the elderly based on our own cases. Discussion whether there is a place for laparoscopy during surgery of the elderly in emergency room (ER) mode. Presentation of the criteria which should be included in the qualification of elderly people before deciding to use surgery or disqualify a patient from such treatment.

\section{Material and methods}

The analysis was made based on the following cases of surgical treatment of elderly patients who were admitted to the hospital in ER mode. Patients were hospitalized in the General and Minimally Invasive Surgery Clinic in Olsztyn in 2016.

\section{Case 1}

An 89-year-old patient admitted to the clinic because of abdominal pain. Preoperative diagnosis showed a tumor on the border of the sigmoid colon and rectum causing bowel obstruction. Comorbidities: hypertension, diabetes mellitus type 2, atrial fibrillation, bronchial asthma, fragility syndrome. The patient had a history of shock. The doctor on duty decided on surgery, using two techniques: excision of the sigmoid colon and end-to-end anastomosis. In the postoperative period seven further procedures were performed because of: anastomotic leak, wound dehiscence, peritonitis, and intestinal fistula. Forty-two units of blood were used, non-adhesive mesh, total parenteral nutrition for 9 months, 8 kinds of antibiotics and antifungal therapy. He was treated for 2 months in the intensive care unit (ICU). Hospitalization time was 9 months and as a result the patient died.

\section{Case 2}

A 65-year-old patient, lying in bed for 30 years, requiring permanent care from others, diagnosed with multiple sclerosis 3 years before, admitted to the clinic because of constipation. Fecal impaction localized in sigmoido-rectal junction, subocclusion. Hartmann's procedure was performed. She was treated with dobutamine, 6 units of blood were used, antibiotics, and antifungal drugs. Hospitalization time was 3 weeks and she died.

\section{Case 3}

An 89-year-old patient, operated on because of adhesive occlusion in a different hospital 9 days before admission. The cause of rehospitalization was wound dehiscence with intestinal fistula. Comorbidities: heart failure, hypertension, bronchial asthma, atherosclerosis. The performed procedure was closure of fistulas with stitches. The patient underwent two operations and received antibiotics and intensive care. The patient died on the $8^{\text {th }}$ postoperative day.

\section{Case 4}

A 75-year-old patient admitted to the clinic because of shock due to gastric hemorrhage after gastroscopy. Comorbidities: heart failure, chronic obstructive pulmonary disease, hypertension, hypercholesterolemia, morbid obesity. Laparotomy was performed, Bll gastric resection. After the operation there was increasing respiratory failure with signs of pneumonia. He was moved to the ICU and on the $2^{\text {nd }}$ postoperative day died. 


\section{Case 5}

A 96-year-old patient, admitted to the clinic because of pyloric stenosis. Comorbidities: myocardial infarction 2 weeks before, frailty syndrome. Antrectomy was performed. He was treated with antibiotics, parenteral nutrition, 6 units of blood, and pleural drainage due to pneumothorax after central venous line. One day before death he was resuscitated and supported alive by noradrenaline.

\section{Case 6}

An 86-year-old patient, admitted to the clinic due to $10 \times 15 \mathrm{~cm}$ irreducible incisional painful hernia after appendectomy. Closure of hernia gate using mesh. After the surgery, there was an enlarging hematoma at the site. On the $1^{\text {st }}$ postoperative day through the wound the hematoma was emptied and the mesh was replaced with a new one to "prevent infection". After the reoperation, the patient choked, was resuscitated and moved to the ICU, where on the $2^{\text {nd }}$ postoperative day he died.

\section{Case 7}

A 77-year-old patient admitted to the clinic two weeks after resection of colorectal cancer with endto-end anastomosis due to malodorous secretion from a postoperative wound. Comorbidities: diabetes mellitus type 2 , heart failure, hypertension, total gastrectomy 5 years before because of cancer. She was treated in the clinic for 34 days with antibiotics, parenteral nutrition, several wound inspections, and blood units. Every single day during the medical round she shouted "let me go, let me die, do not bother me". She died.

\section{Discussion}

In 2015 there were 900 million people aged 60 years and older. According to the WHO by 2050 it is expected to total 2 billion. Nowadays, 125 million people are aged 80 years or older and the number is increasing dramatically [6]. Current demographic trends in Poland are also alarming but should be taken into consideration when planning the development of medical care in the coming years (Figure 1) [7].

Qualification of elderly patients for surgery should take into account not only the physical but also the cognitive status, as well as the comorbidities. The overall status of the patient has to be as- sessed before surgery in order to determine whether the patient is able to tolerate the standard therapy. The aging process leads to some phenotypic changes: there is a gradual loss of height after the thirties as a result of shortening of the joints of lower limbs, deepening of the vertebral curves and compression of the intervertebral discs. After 65 years, changes also occur in body composition, and the level of water decreases. The aging process affects all layers of the skin, with a decrease of the thickness of the skin and loss of its protective qualities, secretion, and perception. For the surgeon, especially important is the loss of mechanical protection and loss of water and electrolytes as well as reducing the perception of stimuli from the environment, such as touch, heat or pain. In the nervous system, the activity of nerve cells and neurotransmitters is decreased, and in the endocrine system there are limitations to the hormonal activity due to reduced hormonal output which triggers the deficiency symptoms. It lowers the body's resistance, both cellular and that mediated by cytokines. This leads to disruption of the functioning of B cells (decreased production of immunoglobulins), T cells (reduced proliferation) and NK cells (reduced cytotoxic activity). The aging of the immune system causes greater susceptibility to infection and a higher incidence of cancer. As a result of the rise of elimination processes and slowing down of proliferation, the number of body cells decreases [1-3].

In old age there are also changes in behavior, particularly short-term memory impairment as a result

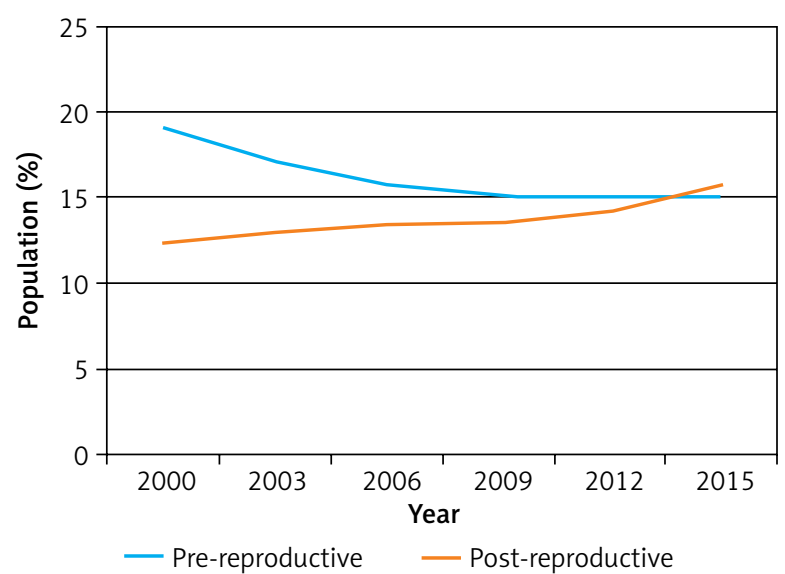

Figure 1. Population development and changes in age structure in 2000-2015 as a percentage of the total population [7] 
of progressive neuronal loss, also decreasing the velocity of nerve conduction, resulting in delayed reactions. This happens as a result of myelination disorders, reduction of the number of ion channels in the neuronal membrane and synaptic dysfunction and an increase in the excitability threshold receptors [8]. All these factors have an impact on the results of surgical treatment, and it should be considered whether, instead of treatment which gives a chance to heal, surgery is not a prelude to permanent medical futility.

One of the most important issues allowing one to determine the state of health of the elderly is frailty syndrome. In the literature, it has been defined as a state of increased vulnerability to poor resolution of homeostasis following stress, which increases the risk of adverse outcomes including falls, delirium and disability. The reserves and resistance to stressors are decreased, resulting from cumulative declines across multiple physiologic systems, and causing vulnerability to adverse outcomes [2]. In 2008 Searle et al. created the 40-variable frailty index as a tool to predict the potential risk of disease [9]. Since then a number of different questionnaires have been developed $[1,10,11]$. Joseph et al. identified the frailty index as an independent predictor of hospital complications and duration of stay of elderly patients undergoing emergency general surgery. Compared with other factors such as the ASA scale, it can be a preoperative assessment tool for surgery [11].

In the elderly, there is significantly reduced ability to adapt to stressful situations, including illness. The result is that a seemingly low level of stress in the form of even minor surgery can be fatal. When opting for surgery in elderly patients, laparoscopy should be taken into account. Laparoscopy leads to a shorter time of convalescence and hospitalization. According to the literature and personal experience, the postoperative pain is lower so patients need fewer painkillers, which disturb their functioning. Because of chronic diseases occurring in the elderly which compromise skin integrity, laparoscopy has an advantage over laparotomy due to better results in wound healing. Also, oral nutrition is provided earlier after minimally invasive surgery than the classic one. Moreover, the risk of postoperative complications is lower after laparoscopy than after open surgery [12].

A large multicenter prospective cohort study of patients who underwent surgery of the upper gas- trointestinal tract clearly showed a marked increase in mortality among patients 65 years old and above. Attention was drawn to several risk factors contributing significantly to increased patient mortality risk: age above 85 years, emergency mode, anemia, leukocytosis, class IV ASA and palliative tumor operations [13].

The cases presented in our study show the results of overzealous treatment in old patients. Six of these patients died as a result of complications after major surgery, and one died during the preparation time. These procedures are typically for younger patients in a good state of health and without major comorbidities. When opting for treatment for the elderly, especially those with frailty syndrome, we should choose a minimally invasive one, which would not radically cure the illness, but would improve functioning and reduce pain and discomfort. Overzealous treatment is also associated with high, unnecessary costs for health care [14]. In choosing the proper procedure for the elderly we should consider the correlation between the costs and the actual usefulness of practice whilst bearing in mind that the tests are performed to take concrete actions, and not to show any senseless step to write in the history of the disease. The daily execution of laboratory tests or imaging in clinically stable patients seems doubtful. Taking into account the statements above, laparoscopy should always be considered in the case of ER procedures or diagnostics.

Besides the standard factors, such as ASA classification and the severity of disease, e.g. the TNM classification, where surgery is considered, the following factors should be taken into account:

1. Comorbidities - diabetes mellitus, hypertension, atherosclerosis, heart failure (NYHA classification), pulmonary disorders, kidney disorders.

2. Mental disorders - degree of independence.

3. Frailty syndrome.

4. Age-related skin changes.

5. The will of the patient.

Surgical treatment of elderly patients should be planned with multidisciplinary teams involving the anesthesiologist and other professionals depending on the indications: neurologist, geriatrician, cardiologist, internist, rehabilitation specialist, psychiatrist, specialist in palliative medicine or nursing [15-17]. Therefore we suggest that geriatric surgery centers should be developed to minimize the risk of overzealous treatment and potential complications. 


\section{Conclusions}

Elderly patients should not be treated as typical adults, but as a separate group of patients requiring special treatment. Due to the existing additional disease in the elderly, the frailty syndrome, any surgical intervention should be minimally invasive. The discussion about therapy should be conducted by a team of specialists from a variety of medical fields. Laparoscopy seems to be the method of choice in the case of ER procedures in the elderly.

\section{Conflict of interest}

The authors declare no conflict of interest.

\section{References}

1. Buckinx F, Rolland Y, Reginster JY, et al. Burden of frailty in the elderly population: perspectives for a public health challenge. Arch Public Health 2015; 73: 19.

2. Clegg A, Young J, lliffe S, et al. Frailty in elderly people. Lancet 2013; 381: 752-62.

3. Walker M, Spivak M, Sebastian M. The impact of aging physiology in critical care. Crit Care Nurs Clin North Am 2014; 26: 7-14.

4. Xynos E, Gouvas N, Triantopoulou C, et al. Clinical practice guidelines for the surgical management of colon cancer: a consensus statement of the Hellenic and Cypriot Colorectal Cancer Study Group by the HeSMO. Ann Gastroenterol 2016; 29: 3-17.

5. Janik M, Stanowski E, Paśnik K. Present status of bariatric surgery in Poland. Videosurgery Miniinv 2016; 11: 22-5.

6. WHO. Ageing and health. Fact sheet N404, September 2015.

7. Central Statistical Office of Poland. Population development and changes in age structure in 1950-2015. Available at http://stat.gov.pl/en/topics/population/population/structureof-the-population-by-2015,7,1.html

8. Singh A, Misra N. Loneliness, depression and sociability in old age. Ind Psychiatry I 2009; 18: 51-5.

9. Searle SD, Mitnitski A, Gahbauer EA, et al. A standard procedure for creating a frailty index. BMC Geriatrics 2008; 8: 24.

10. Sutton JL, Gould RL, Daley S, et al. Psychometric properties of multicomponent tools designed to assess frailty in older adults: a systematic review. BMC Geriatrics 2016; 16: 55.

11. Joseph B, Zangbar B, Pandit V, et al. Emergency general surgery in the elderly: too old or too frail. J Am Coll Surg 2016; 222 805-13.

12. Anania G, Scagliarini L, Santini M, et al. Benefits of laparoscopic colorectal surgery in the geriatric patient. G Chir 2012; 33 352-7.

13. Duron JJ, Duron E, Dugue T. Risk factors for mortality in major digestive surgery in the elderly: a multicenter prospective study. Ann Surg 2011; 254: 375-82.

14. Bock JO, König HH, Brenner $\mathrm{H}$, et al. Associations of frailty with health care costs - results of the ESTHER cohort study. BMC Health Services Research 2016; 16: 128.
15. Ugolini G, Pasini F, Ghignone F, et al. How to select elderly colorectal cancer patients for surgery: a pilot study in an Italian academic medical center. Cancer Biol Med 2015; 12: 302-7.

16. Deiner $S$, Westlake B, Dutton RP, et al. Patterns of surgical care and complications in elderly adults. J Am Geriatr Soc 2014; 62: 829-35.

17. Maffei S, Colantoni A, Kaleci S, et al. Clinical features of colorectal cancer patients in advanced age: a population-based approach. Intern Emerg Med 2016; 11: 191-7.

Received: 24.02.2017, accepted: 15.03.2017. 